\title{
Vínculo de apego, estilos parentales y empatía en adolescentes
}

\author{
Attachment, parental styles and empathy in adolescent
}

\author{
Amelia Paez y Anna Rovella ${ }^{2}$
}

\begin{abstract}
${ }^{1}$ Licenciada en Psicología. Auxiliar de Primera del Área Psicobiológica, Facultad de Psicología (Fapsi), Universidad Nacional de San Luis (UNSL). Becaria doctoral del Consejo Nacional de Investigaciones Científicas y Técnicas (CONICET). Doctoranda de la Facultad de Psicología,

UNSL.E-mail: aepaez@unsl.edu.ar
\end{abstract}

${ }^{2}$ Doctora en Psicología. Profesora Titular en la Cátedra Psicología General, Facultad de Psicología, Universidad Nacional de San Luis (UNSL). Directora de la Maestría en Psicología Clínica, Mención Cognitiva-Integrativa, UNSL. Directora del Laboratorio de Investigaciones en Ciencias del Comportamiento (Fapsi, UNSL). Presidenta de la Asociación Argentina de Ciencias del Comportamiento. E-mail: arovella@unsl.edu.ar

Facultad de Psicología, Universidad Nacional de San Luis.

Ciudad de San Luis, Argentina.

\section{Resumen}

El objetivo de la presente investigación fue estudiar las relaciones entre la percepción del vínculo del apego, de las relaciones parentales y los aspectos cognitivos y emocionales de la empatía en un grupo de adolescentes. Se utilizaron los formatos para madre y padre de las adaptaciones argentinas de la Escala de Seguridad de Kerns y de la versión abreviada del Inventario de la percepción de los hijos acerca de las relaciones con sus padres para adolescentes (CRPBI; subescalas: aceptación, control patológico y autonomía extrema). Se utilizó como medida de empatía, la validación argentina del Índice de Reactividad Interpersonal (componente cognitivo: Toma de Perspectiva y Fantasía; componente emocional: Preocupación Empática y Malestar Personal). Los varones manifestaron menores puntajes totales en el IRI respecto de las mujeres $(\mathrm{p} \leq$ .001). Los adolescentes que experimentaron un apego alto hacia su madre y padre perci- bieron más aceptación en la relación parental con ellos $(p \leq .001 ; p \leq .001)$ y presentaron mayor empatía $(\mathrm{p}=.018 ; \mathrm{p}=.016)$. Al igual que los adolescentes que percibieron un estilo parental caracterizado por la aceptación con su madre y padre $(\mathrm{p}=.008 ; \mathrm{p}=.001)$. Experimentar un apego seguro en la adolescencia podría depender de percibir una relación interpersonal con los progenitores basada en la aceptación de su individualidad e implicación positiva. El establecimiento de un vínculo de apego seguro y percibir la aceptación como práctica parental permite el desarrollo de la empatía.

Palabras clave: Apego; Estilos parentales; Empatía cognitiva; Empatía emocional; Adolescentes.

\section{Abstract}

The aim of this investigation was to study the relationship among the perception of atta- 
chment, parenting relationships, and emotional and cognitive aspects of empathy in a group of adolescents. In order to measure adolescents' perception of the relationship with their parents we used the formats for mother and father of the Argentinean adaptations: (1) Kerns' safety scale, and (2) the abbreviated version of the Children's Report on Parent Behavior (CRPBI); subscales: acceptance, pathological control and extreme autonomy. The Argentine validation of the Interpersonal Reactivity Index (cognitive component: Taking Perspective and Fantasy, emotional component: Empathic Concern and Personal Distress) was used as the empathy measure. Considering the total score obtained on the Kerns safety scale, respect to the maternal and paternal figures, high attachment perception groups (mean $\geq+1 \mathrm{SD}$ ) and low attachment perception groups (mean $\leq-1 \mathrm{SD})$ were formed. Correlations between parental styles and empathy with the Spearman test were evaluated. Mean comparisons were made with the Mann Whitney U test: on the one hand, between gender and empathy; on the other, among adolescents who perceived a low and high attachment with both parents in relation with empathy and parental styles. Males reported lower total IRI scores in the subscales: fantasy, empathic concern and personal distress (respectively: $\mathrm{p} \leq .001 ; \mathrm{p} \leq .001 ; \mathrm{p}$ $\leq .001 ; \mathrm{p} \leq .001)$ as compared to adolescent women. The females that experienced a high attachment towards their parents perceived higher acceptance rate in the relationship with them $(\mathrm{p} \leq .001 ; \mathrm{p} \leq .001)$. They also showed higher empathy $(\mathrm{p}=.018 ; \mathrm{p}=.016)$ and obtained higher scores in the dimensions of the IRI: perspective taking $(\mathrm{p} \leq .001 ; \mathrm{p}=.006)$ and empathic concern $(p=.024 ; p=.022)$. The adolescents who perceived a more tolerant parenting style showed themselves more empathic $(p=.008 ; p=.001)$. In addition, the parental style of extreme autonomy with their mother and their father was negatively associated with the total scores of the IRI ( $p$ $=.006, p \leq .001)$, fantasy $(p=.011 ; p=.017)$ and empathic concern $(\mathrm{p} \leq .001 ; \mathrm{p} \leq .001)$. Parents with less years of schooling use parenting practices based on punitive and coercive control. The female gender was characterized by an empathic emotional response, although both genders have a similar cognitive ability to understand or take the perspective of the other. However, women react more affectively. These differences can be attributed to socialization stereotyped patterns of male and female parenting that assign to women greater emotional sensitivity. Experiencing a secure attachment in adolescence may depend on perceiving an interpersonal relationship with parents based on the acceptance of their individuality and positive involvement. These results match with several investigations, which show that parents perceived as available and with democratic parenting styles, based on the acceptance of adolescents, establish with their children a secure attachment bond, which is associated with a greater psychosocial adjustment. Experiencing a relationship based on acceptance, on affective and committed reciprocity with significant adults that show first empathy and emotional attunement with their children, is a requisite for adolescents to understand the perspective of others and identify with experience, to feel compassion and concern the discomfort of others. Establishing a secure attachment bond and perceiving acceptance as a parental practice allows the development of empathy, protective factor of aggression and promoter of adolescent prosocial behavior.

Keywords: Attachment; Parenting styles; Cognitive empathy; Emotional empathy; Adolescents.

\section{Introducción}

Entre las funciones de la familia, se encuentra la socialización primaria de los hijos desarrollada al inculcarle normas, valores y pautas que permitan su inserción social. Muchas veces la inadecuada socializa- 
ción conduce a actos de vandalismo y agresión, donde priman los intereses individuales en detrimento de los sociales (Mestre-Escrivá, 2014).

A diferencia de los niños y adolescentes que perciben la relación con sus padres basada en la aceptación, aquellos que perciben mayor control, autonomía extrema, indiferencia emocional, rechazo explícito e inconsistencia en la crianza se caracterizan por ser más inestables a nivel emocional, impulsivos, agresivos y con un menor ajuste emocional y comportamental (De la Torre-Cruz, García-Linares y Casanova-Arias, 2014; Oliva, Parra y Arranz, 2008; Richaud de Minzi, 2006a, 2007a;TurPorcar, Mestre-Escrivá, Samper y Malonda, 2012).

En términos generales, la figura de apego sirve como base segura (Ainsworth, Blehar, Waters y Wall, 1978) a partir de la cual el niño experimenta la confianza necesaria para explorar el mundo. Cuando el niño se siente inseguro y no confía en el cuidador, es probable que responda con miedo y ansiedad, o de manera defensiva (Brumariu y Kerns, 2008).

Bowlby (2009) define al apego como cualquier forma de conducta que tiene como resultado el logro o la conservación de otro individuo identificado y concebido como mejor capacitado para enfrentar al mundo. El sistema de apego forma parte de una serie de sistemas conductuales de vinculación que incluyen la exploración, cuidado y apareamiento sexual diseñado para asegurar la supervivencia y procreación. Por lo tanto, es más probable que el niño busque la proximidad del cuidador cuando perciba un peligro en su entorno. La búsqueda de proximidad, la base segura y el refugio seguro son los tres rasgos definitorios y las tres funciones de una relación de apego. El llanto o la sonrisa del niño modifican a través del contagio emocional, las respuestas afectivas y emocionales de sus cuidadores. El estrés del niño genera en sus cuidadores la motivación para actuar antes de que se provoque un evento estresante. Estos lazos emocionales son la base de la empatía en la relación diádica madre/padre-hijo y pueden servir para generar empatía en otros y conseguir ayuda de otros individuos que están por fuera de la diada (Preston y de Waal, 2002).

En este sentido, el contexto familiar es considerado un factor de gran influencia sobre el desarrollo de comportamientos ajustados o desajustados en la adolescencia (Oliva et al., 2008). La familia representa uno de los núcleos determinantes en el desarrollo cognitivo, personal, emocional y socioafectivo de sus hijos, al proporcionarle todas las señales iniciales, como, por ejemplo, si es amado o no, aceptado o rechazado hasta que ingresa en la vida escolar (Samper, Cortés, Mestre-Escrivá, Náchery Tur-Porcar, 2006).

Ciertas prácticas prototípicas de los padres combinadas dan lugar a una serie de estilos de crianza. Entre estos comportamientos se incluyen: la aceptación, el control y el respeto por la individualidad (Richaud de Minzi, 2002).

La aceptación implica compromiso y afecto. Los padres aceptantes se caracterizan por mantener una actitud cálida y sensible a las necesidades de sus hijos. Baumrind (1991) define tres tipos de estilos parentales: democrático, autoritario y permisivo.

Los padres democráticos son cariñosos, flexibles, comunican las reglas con claridad y coherencia, tienen hijos con un mejor ajuste emocional y comportamental (Oliva et al., 2008), así como menor nivel de estrés cotidiano (García-Linares, de la Torre, Carpio, Cerezo y Casanova, 2014) y mayores niveles de felicidad (Rodríguez, Contreras y Merino, 2016).

A diferencia de este estilo, los padres autoritarios refuerzan la rigidez de las normas, son exigentes e insensibles ante las necesidades de los hijos, se enfadan fácilmente y tienden a utilizar un tipo de castigo punitivo y severo. Por último, los padres permisivos-indulgentes se caracterizan por ser laxos e inconsistentes en la disciplina, no refuerzan las reglas y permiten la expresión de impulsos. 
Para Baumrind (1991) los padres que utilizan modelos educativos basados en el autoritarismo, como los que utilizan modelos permisivos-indulgentes con sus hijos, tienden a tener hijos con comportamientos de tipo agresivo e impulsivo. El control implica autoridad, cumplimiento de las demandas y el monitoreo o vigilancia de las conductas de los hijos. Cuando el control deja de ser autoridad para convertirse en dominancia, cuando no se lo utiliza para proteger y cuidar, sino para manipular, se habla de control hostil o patológico (Richaud de Minzi, 2002). En cuanto a la autonomía y la individualidad de los hijos es una práctica que favorece su buen ajuste psicológico; sin embargo, la excesiva autonomía guarda relación con experiencias familiares de evitación y desvinculación afectiva.

En Argentina se han realizado diversos estudios en niños y adolescentes sobre los estilos parentales relacionados a variables como el apego, afrontamiento al estrés, conducta prosocial, empatía, depresión y experiencia de soledad, entre otras (De la Iglesia, Ongaratoy Fernández, 2010; Meiery Oros, 2012; Richaud de Minzi, 2002, 2005a, 2005b, 2006a, 2006b, 2007a, 2007b, 2009).

Meier y Oros (2012) destacan que en los adolescentes, percibir un elevado control patológico como práctica materna, minimiza la experiencia de la tranquilidad, la gratitud y la satisfacción con la vida. Además, un estilo parental materno caracterizado por una elevada autonomía extrema minimiza la expresión de la gratitud. Por otro lado, la percepción de una elevada aceptación paterna junto con un bajo control patológico y una baja autonomía extrema, propician la tranquilidad. Cuando el control que se ejerce sobre los adolescentes es exagerado y está basado en estrategias psicológicas encubiertas, como la culpa y el temor, para conseguir obediencia, se trata de una forma de control patológica (Richaud de Minzi, 2002). Además, la promoción de una autonomía extrema por parte de la madre se asocia a desvinculación e indiferencia afectiva, dificulta la capacidad de los hijos para reconocer y valorar la bondad ajena y para percibirse como receptores de favores $\mathrm{y}$ beneficios.

En cuanto a la seguridad en relación al vínculo de apego y los estilos parentales, Richaud de Minzi (2006a) postula que percibir la aceptación de las madres y los padres se asocia positivamente con el desarrollo de una base de apego segura, es decir, que para los hijos, sentirse seguro de sus progenitores, dependerá del grado de disponibilidad de ellos cuando se los necesita o demanda.

La manifestación de conductas agresivas en la niñez y adolescencia, se encuentra estrechamente vinculada a los estilos de educación y crianza empleados por los padres (Richaud de Minzi, 2007b), a través de los cuales influyen y modelan el desarrollo empático de los niños y adolescentes, promoviendo la prosocialidad (Carlo, Mestre-Escrivá, Samper, Tur-Porcary Armenta, 2010; Mestre-Escrivá, 2014).

Diversos estudios establecen relaciones entre la dimensión afectiva basada en la aceptación y aplicación de normas coherentes durante la crianza, se asocia con la conducta empática y prosocial en los adolescentes. Por el contrario, relaciones caracterizadas por el rechazo hacia el hijo, hostilidad, críticas excesivas, negligencia y permisividad se asocian con conductas agresivas en los adolescentes (Tur-Porcar et al., 2012).

La empatía consiste en la capacidad de ponerse en el lugar del otro y comprenderlo, a partir de lo que se observa, de la información verbal y de la respuesta afectiva de compartir su estado emocional (Eisenberg, 2000). Definida desde una perspectiva multidimensional, toma en cuenta aspectos cognitivos y afectivos.

Diversas investigaciones sugieren diferencias entre varones y mujeres respecto de la empatía (Gorostiaga, Balluerka, y Soroa, 2014; Paez y Ulagnero, 2015).Las mujeres manifiestan una mayor reacción emocional empática, lo cual se asocia con estereotipos de género que responden a normativas culturales, donde las mujeres reciben una crianza 
orientada al bienestar del otro, mientras que los varones reciben una educación orientada a promover su autonomía (Mestre-Escrivá, Samper, Frías y Tur-Porcar, 2009).

La estabilidad emocional constituye un factor fundamental para establecer relaciones empáticas. Los adolescentes con adecuado control de impulsos emocionales manifiestan mayor conformidad social y menos agresión, respetando las normas sociales (Mestre-Escrivá, Tur-Porcar, Samper y Latorre, 2010; Ulagnero et al., 2008). En síntesis, la empatía desempeña una función inhibidora de la conducta agresiva (Del Barrio, Carrasco, Rodríguez y Gordillo, 2009; Mestre-Escrivá, 2014) y favorecedora de la conducta prosocial (Rodríguez, Mesurado, Oñate, Guerra y Menghi, 2017; Ulagnero, Paez, Jofré y De Bortoli, 2008). La conducta prosocial, y las variables cognitivas y emocionales relacionadas, promueven el desarrollo de habilidades sociales y la adaptación a diversos entornos interpersonales (Taylor, Eisenberg, Spinrad, Eggum y Sulik, 2013).

El propósito del presente estudio fue analizar las relaciones entre la percepción del vínculo del apego, de las relaciones parentales y los aspectos cognitivos y emocionales de la empatía en un grupo de adolescentes escolarizados.

El estudio de las relaciones entre el vínculo de apego, estilos parentales y empatía implica un aporte para el ámbito clínico y escolar, ya que su análisis es pertinente para la elaboración de estrategias destinadas a promover el desarrollo de conductas empáticas en adolescentes desde el núcleo familiar, desembocando en la reducción de los niveles de violencia en los diferentes ámbitos de interacción interpersonal.

\section{Método}

\section{Muestra}

La muestra fue intencional con una selección no probabilística. Estuvo conformada por 518 adolescentes que asistían al nivel de estudio secundario de establecimientos educativos públicos de la ciudad de San Luis y de la ciudad de Tunuyán, provincia de Mendoza (Argentina), con un rango de edad entre 13 y 20 años $(\mathrm{M}=15.22 ; \mathrm{DE}=1.69,65 \%$ mujeres).Se excluyeron aquellos participantes con retraso mental, con enfermedad neurológica u orgánica que afectan el funcionamiento cognitivo y los que recibían terapia psicofarmacológica. Además, se excluyeron a quienes no recibieron la autorización de sus padres o tutores y también a aquellos cuyos protocolos estaban deficitarios o incompletos.

\section{Instrumentos}

1.- Versión madre y padre de la Escala de Seguridad de Kerns (Kerns, Klepac y Cole, 1996; adaptación argentina de Richaud de Minzi, Sacchi y Moreno, 2001). Esta escala consta de 18 ítems, con el siguiente formato de enunciación: "Algunos chicos sienten que pueden contar con su mamá/papá cuando la/o necesitan". Cada ítem presenta tres opciones de respuestas: "sí, me parezco", "me parezco en parte", "no me parezco". Mide las percepciones de los niños y adolescentes sobre la seguridad en el vínculo de apego en una dimensión única y continua. Sin embargo, Lieberman, Doyle y Markiewicz (1999) sugirieron una calificación alternativa para obtener dos dimensiones de la seguridad: disponibilidad y confianza en la figura de apego. Un alto puntaje se interpreta como un tipo de apego seguro.

En la validación argentina $(\mathrm{N}=1421)$, se conservaron sólo 10 ítems (5 pertenecientes a la dimensión confianza y 5 a la dimensión disponibilidad) de los 15 ítems de la escala original, y se omitieron los relativos a la calidad de la comunicación, ya que no cumplían con los criterios psicométricos. Las investigaciones han señalado buenas propiedades psicométricas de este instrumento en Argentina, tanto en lo que respecta a la confiabilidad como a la validez (Greco, 2013; 
Richaud de Minzi, 2010). El alfa de Cronbach fue de .81 para la versión madre y de .83 para la versión padre de la escala.

2.- Versión abreviada del Inventario de la percepción de los hijos acerca de las relaciones con sus padres para adolescentes (Richaud de Minzi, 2005b). El inventario consta de 32 ítems en versiones separadas para la madre y para el padre, y se responde eligiendo entre tres opciones: Sí, Algo, No. Esta versión es una adaptación argentina del Children's Report of Parental Behavior Inventory (CRPBI) de Schaefer (1965) para adolescentes de 13 a 17 años, que evalúa las siguientes prácticas parentales desde la percepción de los hijos: (a) control patológico, (b) aceptación y (c) autonomía extrema. El control patológico implica castigo, generación de culpa y ansiedad o el retiro afectivo o de las relaciones, indagado a través de ítems como por ejemplo "Mi mamá/ papá quisiera que me quedara en casa para controlarme". La aceptación consiste en que los progenitores satisfacen las necesidades de sus hijos, que aceptan su autonomía y se involucran positivamente con ellos (ejemplo: "Mi mamá/papá me dice lo mucho que me quiere"). La autonomía extrema o negligencia se caracteriza por no ocuparse o involucrarse en las necesidades del niño y dejar que se arregle solo (ejemplo: "Mi mamá/papá me deja salir cuantas veces quiera"). Así, dentro del factor aceptación se incluyen las subdimensiones aceptación, aceptación de la individualidad, centrado en el niño e implicación positiva, y dentro del factor autonomía extrema se encuentran autonomía extrema y disciplina laxa. El control patológico abarca las subdimensiones control extremo, control hostil, instalación de ansiedad y culpa, retiro de relaciones, rechazo, intrusión y posesividad.

El coeficiente alfa de Cronbach reportado por Richaud de Minzi (2005b) para cada uno de los tres factores, fue de .81 en aceptación, .83 en control patológico y .75 en autonomía extrema, para la versión madre, y de.82 en aceptación, .84 en control patológico y .73 en autonomía extrema, para la del padre. En la presente investigación el alfa de la versión madre para el factor aceptación fue de .855 , para control patológico, .815 y para autonomía extrema .587. Para la versión padre en la dimensión aceptación el alfa fue de .869 , en control patológico .828 y en autonomía extrema .553.

3-Validación argentina para adolescentes (Paez, Ulagnero, Jofré y De Bortoli, 2008) del Índice de Reactividad Interpersonal (IRI) de Mestre-Escrivá et al. (2004). El IRI es un instrumento que evalúa la empatía desde una perspectiva multidimensional que incluye dos factores cognitivos y dos emocionales. Está compuesto por 28 ítems evaluados en escala tipo Likert (1 a 5), distribuidos en cuatro subescalas: Toma de perspectiva, Fantasía, Preocupación empática y Malestar personal. Por un lado, las subescalas Toma de perspectiva y Fantasía miden los aspectos cognitivos de la empatía. Por otro lado, las subescalas Preocupación empática y Malestar personal evalúan la dimensión emocional de las respuestas empáticas (Davis, 1980; Mestre-Escrivá et al., 2004). La subescala Toma de perspectiva incluye ítems que evalúan la habilidad para adoptar o comprender el punto de vista de los demás ante situaciones de la vida cotidiana, por ejemplo: "A menudo tengo sentimientos de ternura y de preocupación hacia la gente menos afortunada que yo". La subescala Fantasía indica la tendencia a identificarse con personajes ficticios (cine, literatura) y la capacidad para imaginarse en situaciones irreales (ejemplo: "Me siento identificado/a con los sentimientos de los personajes de las novelas"). La subescala Preocupación empática mide los sentimientos de compasión, preocupación y cariño al observar el malestar de los demás (ejemplo: "Las desgracias de otras personas, por lo general, no me perturban mucho"). La escala de Malestar personal evalúala ansiedad y el malestar ante situaciones negativas de otras personas (ejemplo: "En las situaciones de emergencia me siento incómodo/a y temeroso/a"). 
La consistencia interna del instrumento reportada en adolescentes argentinos por Paez et al. (2008) indicó para el IRI (puntaje total) un alfa de Cronbach de .736; para Toma de perspectiva .695, para Fantasía .737, para Malestar personal .657 y para Preocupación empática .479 .

El alfa de Cronbach de la presente investigación indicó valores de.654 para el puntaje total de la escala, .661 para Toma de perspectiva, .660 para Fantasía, .526 para Malestar personal y .441 para Preocupación empática.

\section{Procedimiento}

Los adolescentes fueron informados sobre los objetivos, las finalidades de la investigación y el carácter confidencial de los datos. Posteriormente, se solicitó por escrito el consentimiento voluntario de los progenitores o tutores a cargo.

Las pruebas fueron administradas durante el horario escolar, en dos sesiones de 45 minutos, por miembros del equipo de investigación capacitados en el manejo de los instrumentos.

\section{Análisis de datos}

Se realizó un análisis estadístico descriptivo e inferencial y de distribución normal de la muestra con la prueba de Kolmogorov-Smirnov. Se evaluaron las correlaciones entre estilos parentales y empatía con la prueba de Spearman. A partir del puntaje total obtenido en la escala de seguridad de Kerns, se conformaron los grupos de percepción de apego alto y bajo respecto a la figura materna y paterna. El grupo de percepción de apego materno alto se conformó por aquellos adolescentes cuyos puntajes totales en la versión madre de la Escala de Kerns fueron superiores a 1DE y el grupo de percepción de apego materno bajo se constituyó con los participantes cuyos puntajes totales de la versión madre de la Escala de Seguridad de Kerns fueron inferiores a 1DE. El mismo procedimiento se aplicó para determinar los grupos de apego paterno alto y apego paterno bajo. Con la prueba $U$ de Mann Withney se compararon, entre los grupos de percepción de apego alto y bajo (madre y padre), por un lado, los niveles de empatía, y por otro, los estilos parentales (aceptación, control patológico y autonomía).

\section{Resultados}

\section{Análisis de la distribución muestral}

Al analizar la distribución muestral con la prueba de Kolmogorov-Smirnov, se observó que no presentaron una distribución normal las variables apego con sus respectivas dimensiones, disponibilidad y confianza, el CRPBI y las prácticas de crianza parentales (autonomía extrema, aceptación y control patológico) y el IRI y sus subescalas (Toma de perspectiva, Fantasía, Preocupación empática y Malestar personal).

\section{Estadística descriptiva de apego, CRPBI e IRI}

En cuanto a la distribución de acuerdo a la percepción del apego hacia la madre, el $17 \%$ presentó una percepción baja del apego y el $15 \%$ percibió un alto apego. En relación al vínculo de apego con el padre, el $17.3 \%$ de los adolescentes percibió un apego bajo y el $18.7 \%$, un apego alto.

En la Tabla 1 se muestran los valores descriptivos para ambos progenitores de la variable apego (Escala de Seguridad de Kerns) y del CRPBI. 
Tabla 1. Estadística descriptiva de apego y del CRPBI, versión madre y padre.

\begin{tabular}{cccccc} 
& N & Mín. & Máx. & M & DE \\
\cline { 2 - 6 } MADRE & & & & & \\
\hline Apego (puntaje total) & 491 & 3 & 73 & 45.41 & 6.684 \\
Confianza & 491 & 3 & 35 & 23.05 & 3.321 \\
Disponibilidad & 490 & 7 & 52 & 22.40 & 3.995 \\
CRPBI (puntaje total) & 438 & 31 & 94 & 65.09 & 7.929 \\
Aceptación & 438 & 7 & 24 & 19.36 & 4.114 \\
Control Patológico & 393 & 6 & 52 & 29.21 & 6.998 \\
Autonomía Extrema & 438 & 7 & 24 & 14.41 & 3.132 \\
\hline PADRE & & & & & \\
CRPBI (puntaje total) & 393 & 10 & 94 & 60.79 & 9.172 \\
Aceptación & 393 & 3 & 24 & 17.52 & 4.499 \\
Control Patológico & 393 & 6 & 52 & 29.21 & 6.998 \\
Autonomía Extrema & 393 & 1 & 20 & 12.66 & 2.810 \\
Apego (puntaje total) & 475 & 8 & 73 & 44.52 & 6.986 \\
Confianza & 475 & 5 & 30 & 22.76 & 3.335 \\
Disponibilidad & 475 & 3 & 52 & 21.76 & 4.358 \\
\hline
\end{tabular}

En la Tabla 2 se presentan los valores descriptivos de las dimensiones de la empatía evaluada con el IRI.

Tabla 2. Estadística descriptiva del IRI y de sus sub-escalas PT, FS, EC y PD.

\begin{tabular}{ccccc}
\hline & Mín. & Máx. & M & DE \\
\hline IRI (puntaje total) & 46 & 144 & 85.53 & 14.032 \\
Toma de Perspectiva & 8 & 35 & 20.93 & 4.846 \\
Fantasía & 9 & 37 & 21.19 & 5.334 \\
Preocupación Empática & 7 & 90 & 24.39 & 6.025 \\
Malestar Personal & 6 & 36 & 19.02 & 5.153 \\
\hline
\end{tabular}

\section{Género y empatía}

En relación a las diferencias de media en empatía según el género (Tabla 3), las mujeres se manifestaron más empáticas al obtener un mayor puntaje total en el IRI y en las subescalas Fantasía, Preocupación empática y Malestar personal en comparación con los varones $(p \leq .001$ en todos los casos). 
Tabla 3. Diferencias de medias en empatía entre varones y mujeres.

\begin{tabular}{ccccccc} 
& Sexo & $\mathrm{N}$ & $M$ & $D E$ & $\mathrm{z}$ & $\mathrm{p}$ \\
\hline IRI (puntaje total) & $\mathrm{F}$ & 308 & 87.44 & 13.935 & -4.379 & .000 \\
& $\mathrm{M}$ & 153 & 81.32 & 13.173 & & \\
Toma de perspectiva & $\mathrm{F}$ & 308 & 20.82 & 4.789 & -.414 & .679 \\
& $\mathrm{M}$ & 153 & 21.06 & 4.970 & & \\
Fantasía & $\mathrm{F}$ & 308 & 21.79 & 5.489 & -3.498 & .000 \\
Preocupación empática & $\mathrm{M}$ & 153 & 19.87 & 4.758 & & \\
Malestar personal & $\mathrm{F}$ & 308 & 25.11 & 6.129 & -3.693 & .000 \\
& $\mathrm{M}$ & 153 & 22.89 & 5.600 & & \\
& $\mathrm{~F}$ & 308 & 19.72 & 5.198 & -4.286 & .000
\end{tabular}

Diferencias de medias en empatía entre mujeres con apego alto y bajo (materno y paterno).

Al analizarse las diferencias de medias entre las adolescentes con apego alto y bajo hacia su madre, el primer grupo manifestó una mayor toma de perspectiva $(\mathrm{p}=.004)$ respecto de las que presentaban un apego bajo. En cuanto a la figura paterna, no se hallaron diferencias significativas entre los grupos de mujeres con apego alto y bajo en relación a las subescalas del IRI.

\section{Análisis correlacional entre estilos} parentales (materno y paterno) y empatía en el grupo de mujeres.

En las adolescentes, percibir un estilo parental materno de aceptación se asoció positivamente con un mayor puntaje total en el IRI y en las subescalas Toma de perspectiva y Fantasía (respectivamente, $r=.152$, $p=.004 ; r=.137, p=.009 ; r=.136$, $p=.009)$. La práctica materna de control patológico correlacionó positivamente con mayores puntajes totales en el IRI y en la subescala Malestar personal $(r=.112$, $p=.042 ; r=.207, p \leq .001)$. Por último, la autonomía extrema como práctica materna se asoció negativamente con fantasía $(r=-.117$, $p=.026)$.

Apego, estilos parentales y empatía en varones. Diferencias de medias en empatía entre varones con apego alto $\mathbf{y}$ bajo (materno y paterno).

Respecto al grupo de varones, aquellos que percibieron un apego materno alto manifestaron un mayor puntaje promedio en preocupación empática que los que experimentaron un apego materno bajo $(\mathrm{p}=.018)$. Por otro lado, en los varones que experimentaron un apego paterno alto se observó un mayor puntaje total del IRI y en la subescala Preocupación empática $(\mathrm{p}=.038, \mathrm{p}=.044)$.

\section{Análisis correlacional entre estilos parentales (materno y paterno) y empatía en el grupo de varones.}

En el grupo de varones, al correlacionarse las variables práctica de crianza materna y empatía, se observó por un lado, que la aceptación materna se asoció positivamente con la preocupación empática $(\mathrm{r}=.163, \mathrm{p}=.024)$. Por otro lado, la práctica paterna de aceptación correlacionó positivamente con la toma de perspectiva $(\mathrm{r}=.158, \mathrm{p}=.038)$. 
Diferencias de medias en estilos parentales entre el total de adolescentes con apego alto y bajo

Al compararse la percepción del apego (bajo y alto) con las prácticas parentales, se determinó la existencia de diferencias estadísticamente significativas entre el grupo de adolescentes que perciben un nivel de apego alto con su madre respecto de la práctica materna Aceptación evaluada con el CRPBI $(z=-7.093, p \leq .001)$. Así, los valores de las medias indican que el grupo con un apego alto hacia sus madres perciben más aceptación en la relación con ellas $(M=21.01 ; D E=2.87)$, que aquellos adolescentes con apego bajo $(M$ $=15.62 ; D E=5.07)$.

Los adolescentes con apego alto hacia su padre percibieron una relación con él basada en la aceptación $(z=-6.614 ; p \leq .001)$ en comparación con el grupo con apego bajo.

\section{Análisis correlacional entre apego y estilos parentales}

Además, el análisis correlacional con la prueba de Spearman, indicó que a mayor calidad en el vínculo de apego (confianza y disponibilidad) con la madre $(r=.375 ; p \leq .001)$ y con el padre $(r=.377 ; p \leq .001)$, aumenta la percepción de una relación parental basada en la aceptación con ambos progenitores.

\section{Diferencias de medias en empatía entre adolescentes con apego alto y bajo}

$\mathrm{Al}$ analizarse las diferencias entre las percepciones de apego materno (alto y bajo) y empatía, el grupo que percibió baja calidad en el vínculo de apego materno presentó menor nivel de empatía $(p=.018)$, de toma de perspectiva $(p \leq .001)$ y de preocupación empática $(p=.024)$ que el grupo con apego alto hacia sus madres (Tabla 4).

Tabla 4. Diferencias de medias en empatía entre adolescentes con apego materno bajo y alto.

\begin{tabular}{ccccccc} 
& $\begin{array}{c}\text { Percepción de } \\
\text { apego materna }\end{array}$ & $\mathrm{N}$ & $M$ & $D E$ & $\mathrm{z}$ & $p$ \\
\hline \multirow{2}{*}{ IRI (puntaje total) } & Apego bajo & 92 & 82.39 & 14.903 & -2.367 & .018 \\
& Apego alto & 98 & 86.86 & 13.164 & & \\
Toma de perspectiva & Apego bajo & 92 & 19.87 & 4.363 & -3.552 & .000 \\
& Apego alto & 98 & 22.13 & 4.558 & & \\
Fantasía & Apego bajo & 92 & 20.43 & 5.313 & -1.226 & .220 \\
& Apego alto & 98 & 21.41 & 5.466 & & \\
Preocupación empática & Apego bajo & 92 & 22.57 & 5.391 & -2.254 & .024 \\
& Apego alto & 98 & 24.43 & 5.487 & & \\
Malestar personal & Apego bajo & 92 & 19.52 & 5.200 & -.717 & .473 \\
& Apego alto & 98 & 18.89 & 5.056 & & \\
\hline
\end{tabular}

Al igual que los resultados obtenidos con el nivel de percepción del apego materno y la empatía, los adolescentes cuyo apego hacia el padre fue bajo manifestaron una menor empatía $(p=.016)$, toma de perspectiva $(p=$ $.006)$ y preocupación empática $(p=.022)$ que los adolescentes con un nivel de apego alto hacia su padre (Tabla 5). 
Tabla 5. Diferencias de medias en empatía entre adolescentes con apego paterno bajo y alto.

\begin{tabular}{ccccccc} 
& $\begin{array}{c}\text { Percepción de } \\
\text { apego paterna }\end{array}$ & $\mathrm{N}$ & $M$ & $D E$ & $\mathrm{z}$ & $p$ \\
\hline IRI (puntaje total) & Apego bajo & 88 & 83.44 & 14.280 & -2.403 & .016 \\
& Apego alto & 123 & 88.03 & 13.942 & & \\
Toma de Perspectiva & Apego bajo & 88 & 20.64 & 4.488 & -2.757 & .006 \\
& Apego alto & 123 & 22.31 & 4.898 & & \\
Fantasía & Apego bajo & 88 & 20.53 & 5.137 & -1.540 & .124 \\
& Apego alto & 123 & 21.81 & 5.755 & & \\
Preocupación & Apego bajo & 88 & 23.00 & 5.072 & -2.299 & .022 \\
Empática & Apego alto & 123 & 24.78 & 5.459 & & \\
Malestar Personal & Apego bajo & 88 & 19.27 & 5.347 & -.037 & .971 \\
& Apego alto & 123 & 19.13 & 5.136 & & \\
\hline
\end{tabular}

\section{Análisis correlacional entre estilos parentales y empatía}

En cuanto al análisis de las correlaciones entre las prácticas de crianza parentales y la empatía, en la relación con la madre, los adolescentes que obtuvieron mayores puntajes totales en el IRI, en toma de perspectiva, fantasía y preocupación empática percibieron una mayor aceptación por parte de sus madres (respectivamente: $r=.130, p=.008 ; r=.133$, $p=.006 ; r=.103, p=.034)$. Además, la práctica parental autonomía extrema se asoció negativamente con los puntajes totales del IRI, fantasía y preocupación empática (respectivamente: $r=-.133, p=.006 ; r=-.123, p=.011$; $r=-.171 ; p \leq .001)$ y el malestar personal correlacionó positivamente con la percepción de control patológico en la relación materna $(r$ $=.166 ; p=.001)$.

En cuanto a la relación con el padre, los adolescentes que obtuvieron mayores puntajes en toma de perspectiva percibieron una mayor aceptación como práctica paterna (respectivamente: $r=.171, p=.001)$. Además, la práctica de crianza autonomía extrema se asoció negativamente con los puntajes totales del IRI y con las subescalas Fantasía y Preocupación empática (respectivamente: $r=-.182, p \leq .001$; $r=-.122, p=.017 ; r=-.213 ; p \leq .001)$ y el malestar personal se asoció positivamente con la percepción de control patológico $(r=.166$; $p=.001)$.

\section{Discusión}

En cuanto a la relación entre las dimensiones de la empatía y el género, los resultados del presente estudio concuerdan con los obtenidos en investigaciones en las que se ha observado que las mujeres obtienen puntuaciones más elevadas que los varones en este constructo (Gorostiaga et al., 2014; Mestre-Escrivá et al., 2002, 2004; Mesurado et al., 2014; Rose y Rudolph, 2006; Sánchez-Queija, Oliva y Parra, 2006). A partir de estos resultados, se conjetura que el género femenino se caracteriza por una respuesta emocional empática, y aunque ambos géneros tienen una capacidad cognitiva similar para comprender o tomar la perspectiva del otro, las mujeres reaccionan más afectivamente (Mestre-Escrivá et al., 2009). Estas diferencias pueden ser atribuidas a las divergencias en las pautas de crianza de hombres y mujeres guiadas por los estereotipos sociales, que atribuyen a la mujer mayor sensibilidad emocional, cuidados propios del rol materno y mayor orientación interpersonal respecto de los varones (Garaigordobil y Maganto, 2011). 
En relación al vínculo de apego y los estilos parentales, a partir de los datos obtenidos se infiere que en la adolescencia, experimentar un apego seguro con ambos progenitores se asocia a percibir una relación parental, basada en la aceptación de la individualidad e implicación positiva (Gallarin y Alonso-Arbiol, 2012).

Los adolescentes que perciben a sus progenitores como una base de apego segura, disponible emocionalmente y confiable cuando se la necesita o demanda, experimentan una relación con ellos centrada en la aceptación, caracterizada por mantener una actitud cálida y sensible a las necesidades de sus hijos. Estos resultados coinciden con diversas investigaciones, donde se evidencia que los padres percibidos como disponibles y con estilos de crianza democráticos, en los cuales se destaca la aceptación, la implicancia positiva y normas de conductas claras, establecen con sus hijos un vínculo de apego seguro, el cual se asocia con un mayor ajuste psicosocial, sentimientos de seguridad y estrategias de afrontamiento más adaptativas (Brumariu, Obsuthy LyonsRuth, 2013; Lecannelier, Ascanio, Flores y Hoffmann, 2011; Richaud de Minzi, 2006a, 2006b; Richaud de Minzi, 2007a).

Los adolescentes que manifestaron una mayor capacidad empática percibieron a sus padres como una base de apego segura y una relación en base a la aceptación de los adolescentes y sus experiencias afectivas. Richaud de Minzi (2009) afirma que la empatía requiere, para su desarrollo, de una relación cercana, afectiva y comprometida con adultos significativos que muestren primero empatía y sintonización emocional con los hijos. Al observar y vivenciar conductas empáticas por parte de sus padres, los adolescentes incorporan, mediante modelado, estos comportamientos. Es decir, el establecimiento de una relación de apego donde los adolescentes puedan expresar sus sentimientos y vivenciar la reciprocidad afectiva de sus padres, contribuirá al aprendizaje de conductas empáticas. La internalización de un vínculo de apego seguro incide en la capacidad de regular las emociones promoviendo el establecimiento de relaciones interpersonales ajustadas durante la adolescencia y a lo largo de los cambios del ciclo vital (Balabanian, Lemos y Vargas Rubilar, 2015; Oliva, 2011; Otiz et al., 1993).

Percibir durante la adolescencia prácticas parentales de aceptación, dimensión que conforma el estilo parental autorizativo o democrático, permite desarrollar una mayor capacidad empática, en cuanto a la comprensión de la perspectiva e identificación con la experiencia, a sentir compasión y preocupación ante el malestar ajeno. Por otro lado, en el presente estudio aquellos adolescentes que percibieron en ambos progenitores la autonomía extrema o negligencia como práctica parental manifestaron una menor capacidad empática. Estos resultados expuestos siguen la línea de investigaciones como la de Tur-Porcary colaboradores (2012), quienes concluyen que los factores de crianza punitivos, de hostilidad, negligencia y permisividad se relacionan positivamente con la agresividad de los hijos adolescentes. Los padres pueden estimular la capacidad empática a través del modelado y la implementación de pautas de disciplina con orientación afectiva, que permitan a sus hijos entender y experimentar preocupación ante el malestar de otros. En este sentido, la autonomía extrema o indiferencia parental genera en sus hijos indiferencia hacia las demás personas (Richaud de Minzi, Lemos y Mesurado, 2011).

De la Torre-Cruz y colaboradores(2014) realizaron en España un estudio de tipo correlacional sobre las relaciones entre estilos educativos parentales y agresividad en adolescentes, comprobando que la percepción de un estilo democrático se asocia a un menor grado de expresión de conducta agresiva e indicando que un estilo parental caracterizado por el apoyo, la aceptación e implicación positiva promueve el desarrollo de empatía, conducta predictora de la prosocialidad y amortiguadora de la agresión (Sánchez-Queija et al., 2006). En este sentido, Senabre, Ruiz y Murgui 
(2012) indican que percibir un estilo educativo democrático, que combina altos niveles de afecto y comunicación junto con aplicación de normas y pautas de comportamiento, está vinculado con menores niveles de agresión durante la adolescencia.

La presente investigación realiza aportaciones sobre los estilos parentales que resultan más relevantes en el estudio de la empatía, contribuyendo en el diseño de programas psicoeducativos de intervención en adolescentes y padres, destinados a fomentar el establecimiento de un apego seguro, de relaciones paterno-filiales positivas que promuevan, desde el núcleo familiar, el desarrollo de la empatía como factor protector de la violencia y promotor de la prosocialidad. De acuerdo a lo esperado, y en coincidencia con lo reportado por la literatura científica, el estilo caracterizado por la aceptación de ambos progenitores estimula el desarrollo de una conducta empática frente al estilo caracterizado por la autonomía extrema. Además, en el presente estudio se esperaba hallar que el estilo caracterizado por el control patológico se asociara a menores niveles de empatía en los adolescentes. Posiblemente, estos resultados se deban a la limitación de contar únicamente con la perspectiva de los adolescentes sobre la relación con sus padres y el carácter transversal del estudio, el cual no permite determinar la posible relación causal entre las variables estudiadas. Al margen de dichas limitaciones, este estudio implica un aporte a nivel académico y clínico sobre el papel que juega la calidad del vínculo de apego y la aceptación como práctica de crianza en el desarrollo de la empatía durante la adolescencia.

\section{Referencias bibliográficas}

Ainsworth, M.P., Blehar, M., Waters, E. y Wall, S. (1978). Patterns of attachment.Hillsdale, NJ: Njerbaum.

Balabanian, C., Lemos, V. y Vargas Rubilar, J. (2015). Apego percibido y conducta proso- cial en adolescentes. Revista Colombiana de Ciencias Sociales, 6(2), 278-294. doi: 10.21501/22161201.1515

Baumrind, D. (1991). Parenting styles and adolescent development. En J. Brooks-Gunn, R. Lerner, y A.C. Petersen (Eds.), The encyclopedia of adolescence. New York: Garland.

Bowlby, J. (2009). Una base segura. Aplicaciones Clínicas de una teoría del apego. Buenos Aires: Paidós.

Brumariu, L. y Kerns, K. (2008). Mother-child attachment and social anxiety symptoms in middle childhood. Journal of Applied Developmental Psychology, 29, 393-402. doi: 10.1016/j.appdev.2008.06.002

Brumariu, L.E., Obsuth, I. y Lyons-Ruth, K. (2013). Quality of attachment relationships and peer relationship dysfunction among late adolescents with and without anxiety disorders. Journal of Anxiety Disorders, 27, 116-124. doi: 10.1016/j.janxdis.2012.09.002

Carlo, G., Mestre-Escrivá, M.V., Samper, P.,TurPorcar, A. y Armenta, B.E. (2010). The longitudinal relations among dimensions of parenting styles, sympathy, prosocial moral reasoning, and prosocial behaviors. International Journal of Behavioral Development, 35(2), 116-124. doi: 10.1177/0165025410375921

Davis, M. (1980). A multidimensional approach to individual differences in empathy. Catalog of Selected Documents in Psychology, 10(85), 1-17.

De la Iglesia, G., Ongarato, P. y Fernández, M. (2010). Propiedades psicométricas de una Escala de Estilos Parentales e Inconsistencia Parental Percibida (EPIPP). Evaluar, 10, 32-52.

De la Torre-Cruz, M., García-Linares, M. y Casanova-Arias, P. (2014). Relaciones entre estilos educativos parentales y agresividad en adolescentes. Electronic Journal of Research in Educational Psychology, 12(32), 147-170. doi: 10.14204/ejrep.32.13118

Del Barrio, V., Carrasco, M.A., Rodríguez, M.A. y Gordillo, R. (2009). Prevención de la agresión en la infancia y la adolescencia. International Journal of Psychology and Psychological Therapy, 9(1), 101-107. 
Eisenberg, N. (2000). Emotion, regulation, and moral development. Annual Review of Psychology, 51(1), 665-697. doi: 10.1146/annurev. psych.51.1.665

Gallarin, M. y Alonso-Arbiol, I. (2012). Parenting practices, parental attachment and aggressiveness in adolescence: A predictive model. Journal of Adolescence, 35, 1601-1610. doi: 10.1016/j.adolescence.2012.07.002

García-Linares, M.C., de la Torre, M.J., Carpio, M. de la V., Cerezo, M.T. y Casanova, P.F. (2014). Consistencia/inconsistencia en los estilos educativos de padres y madres, y estrés cotidiano en la adolescencia. Revista de Psicodidáctica, 19(2), 307-325. doi: 10.1387/RevPsicodidact. 7219

Gorostiaga, A., Balluerka, N. y Soroa, G. (2014). Evaluación de la empatía en el ámbito educativo y su relación con la inteligencia emocional. Revista de Educación, 364, 12-38.

Garaigordobil, M. y Maganto, C. (2011). Empatía y resolución de conflictos durante la infancia y la adolescencia. Revista Latinoamericana de Psicología, 43(2), 255-266.

Greco, C. (2013). Apego y percepción de felicidad en la mediana infancia: una aproximación a su estudio. Revista de Psicología, 9(17), 105-116.

Kerns, K.A., Klepac, L., y Cole, A.K. (1996). Peer relationships and preadolescents' perceptions of security in the mother-child relationships. Developmental Psychology, 32, 457-466.

Lecannelier, F., Ascanio, L., Flores, F. y Hoffmann, M. (2011). Apego y psicopatología: Una revisión actualizada sobre los modelos etiológicos parentales del apego desorganizado. Terapia Psicológica, 29(1), 107-116.

Lieberman, M., Doyle, A. y Markiewicz, D. (1999). Developmental patterns in security of attachment to mother and father in late childhood and early adolescence: Associations with peer relations. Child Development, 70(1), 202-213. doi: 10.1111/1467-8624.00015

Meier, L.K. y Oros, L.B. (2012). Percepción de las prácticas parentales y experiencia de emociones positivas en adolescentes. Revista de Psicología, 8(16), 73-84.

Mesurado, M., Richaud, M.C., Mestre-Escrivá,
V., Samper-García, P., Tur-Porcar, A., Morales, S. y Viveros, E.F. (2014). Parental expectations and prosocial behavior of adolescents from low-income backgrounds: A cross-cultural comparison between three countries-Argentina Colombia, and Spain. Journal of Cross-Cultural Psychology, 1-18. doi: 10.1177/0022022114542284

Mestre-Escrivá, M.V., Samper García, P.y Frías Navarro, M.D. (2002). Procesos cognitivos y emocionales predictores de la conducta prosocial y agresiva: La empatía como factor modulador. Psicothema, 14(2), 227-232.

Mestre-Escrivá, V., Frías Navarro, M.D. y Samper García, P. (2004). La medida de la empatía: análisis del Interpersonal Reactivity Index. Psicothema, 16(002), 255-260.

Mestre-Escrivá,V., Tur-Porcar, A. y Del Barrio, M.V. (2004). Temperamento y crianza en la construcción de la personalidad. Conducta agresiva, inestabilidad y prosociabilidad. Acción Psicológica, 3(1), 7-20.

Mestre-Escrivá, M.V., Samper, P., Frías, M.D. y Tur-Porcar, A.M. (2009). Are women more empathetic than men? A longitudinal study in adolescence. The Spanish Journal of Psychology, 12(1), 76-83. doi: 10.1017/ S1138741600001499

Mestre-Escrivá, M.V., Tur-Porcar, A., Samper, P. y Latorre, A. (2010). Inestabilidad emocional y agresividad. Factores predictores. Ansiedad y Estrés, 16(1), 33-45.

Mestre-Escrivá, V. (2014). Desarrollo prosocial: crianza y escuela. Revista Mexicana de Investigación en Psicología, 6(2), 115-134.

Oliva, A., Parra, A. y Arranz, E. (2008). Estilos relacionales parentales y ajuste adolescente. Infancia y Aprendizaje, 31, 93-106. doi: 10.1174/021037008783487093

Oliva, A. (2011). Apego en la adolescencia. Acción Psicológica, 8(2), 55-65.

Otiz, M.J., Apodaka, P., Etxeberria, I., Ezeiza, A., Fuentes, M.J. y López, F. (1993). Algunos predictores de la conducta prosocial-altruista en la infancia: empatía, toma de perspectiva, apego, modelos parentales, disciplina familiar e imagen del ser humano. Revista de Psico- 
logía Social, 8(1), 83-98.

Paez, A.E., Ulagnero, C.A., Jofré, M.J. y De Bortoli, M.A. (marzo de 2008). Análisis de la estructura y consistencia interna de la adaptación española del Índice de Reactividad Interpersonal en alumnos de segundo y tercer año de polimodal. Póster presentado en el $I I I$ Encuentro Interamericano de Salud Mental. Ciudad Autónoma de Buenos Aires, Argentina.

Paez, A. y Ulagnero, C. (2015). Personalidad y empatía en adolescentes. En V.A. Martínez-Nuñez, P. Godoy Ponce, M.A. Piñeda, M.B. Fantín, M. Cuello Pagnone, L. Bower, N. De Andrea, E. González, N. Katzer y E. Lucero Morales (Comps.), Avances y Desafios para la Psicología (199-207). San Luis: Nueva Editorial Universitaria.

Preston, S.D. y de Waal, F.B.M. (2002). Empathy: Its ultimate and proximate bases. Behavioral \& Brain Sciences, 25(1), 1-20. doi:10.1017/ S0140525X02000018

Richaud de Minzi, M.C., Sacchi, C., y Moreno, J.E. (2001). Tipos de influencia parental, socialización y afrontamiento de la amenaza en la infancia. Primer Informe del Subsidio de la Agencia Nacional de Ciencia y Tecnología y del Consejo Nacional de Investigaciones Científicas y Técnicas - PICT 1999 04-06300.

Richaud de Minzi, M.C. (2002). Inventario acerca de la percepción que tienen los niños y las niñas de las relaciones con sus padres y madres. Versión para 4 a 6 años. Revista Interamericana de Psicología, 36(1-2), 149-165.

Richaud de Minzi, M.C. (2005a). Estilos parentales y estrategias de afrontamiento en niños. Revista Latinoamericana de Psicología, 37, 47-58.

Richaud de Minzi, M.C. (2005b). Versión abreviada del Inventario de la percepción de los hijos acerca de las relaciones con sus padres para adolescentes. Psicodiagnosticar, 15, 99-106.

Richaud de Minzi, M.C. (2006a).Evaluación del afrontamiento en niños de 8 a 12 años. Revista Mexicana de Psicología, 23(2), 193-201.

Richaud de Minzi, M.C. (2006b). Loneliness and depression in middle and late childhood: The relationship to attachment and parental styles. The Journal of Genetic Psychology, 167(2), 189-210.

Richaud de Minzi, M.C. (2007a). La percepción de estilos de relación con su padre y madre en niños y niñas de 8 a 12 años. Revista Iberoamericana de Evaluación Psicológica, 1(23), 63-81.

Richaud de Minzi, M.C. (2007b). Parental styles and attachment in relation with self control, social skills and coping in children at risk for poverty. En D.M. Devor (Ed.). New developments in parent-child relations (87-110). Hauppauge, New York: Nova Editorial Publishers, Inc.

Richaud de Minzi, M.C. (2009). Influencia del modelado de los padres sobre el desarrollo del razonamiento prosocial en los/las niños/as. Revista Interamericana de Psicología, 43(1), 187-198.

Richaud de Minzi, M.C. (2010). Gender and cultural patterns of mothers' and fathers' attachment and links with children's self-competence, depression and loneliness in middle and late childhood. Early Child Development and Care, 180(1y2), 193-209. doi: 10.1080/03004430903415056

Richaud de Minzi, M.C., Lemos, V. y Mesurado, B. (2011). Relaciones entre la percepción que tienen los niños de los estilos de relación y de la empatía de los padres y la conducta prosocial en la niñez media y tardía. Avances en Psicología Latinoamericana, 29(2), 330-343.

Rodríguez, T.C., Contreras, K. y Merino, C. (2016). Percepción de los estilos de crianza y felicidad en adolescentes y jóvenes de Lima Metropolitana. Perspectiva de Familia, 1, 11-22.

Rodríguez, L.M., Mesurado, B., Oñate, M.E., Guerra, P. y Menghi, M.S. (2017). Adaptación de la Escala de Prosocialidad de Caprara en adolescentes argentinos. Evaluar, 17(2), 177-187.

Rose, A.J. y Rudolph, K.D. (2006). A review of sex differences in peer relationship processes: Potential trade-offs for the emotional and behavioral development of girls and boys. Psycholo- 
gical Bulletin, 132, 98-131. doi: 10.1037/00332909.132.1.98

Senabre, P., Ruiz, Y. y Murgui, S. (2012).Estilos de parentalidad y su relación con la conducta agresiva. Edetania, 42, 145-157.

Sánchez-Queija, I., Oliva, A. y Parra, A. (2006). Empatía y conducta prosocial durante la adolescencia. Revista de Psicología Social, 21(3), 259-271.

Samper, P., Cortés, M.T., Mestre-Escrivá, V., Nácher, M.J. y Tur-Porcar, A.M. (2006). Adaptación del Child's Report of Parent Behavior Inventory a población española. Psicothema, 18(2), 263-271.

Schaefer, E.S. (1965). Children's Reports of Parental Behavior: an inventory. Child Development, 36, 413-424.

Taylor, Z., Eisenberg, N., Spinrad, L., Eggum, N. y Sulik, M. (2013).The relations of ego-resiliency and emotion socialization to the development of empathy and prosocial behavior across early childhood.Emotion,13(5),822831.doi: 10.1037/a0032894

Tur-Porcar, A., Mestre-Escrivá, V., Samper, P. y Malonda, E. (2012). Crianza y agresividad de los menores: ¿es diferente la influencia del padre y de la madre? Psicothema, 24, 284-288.

Ulagnero, C.A., Paez, A.E., Jofré, M.J. y De Bortoli, M. (2008). Estilos de personalidad y empatía en adolescentes de segundo y tercer año de polimodal. En A. Timboli, J.C. Fantin, S. Raggi, P. Fridman, E. Grande y G. Betran (Comps.). Modernidad, tecnología y síntomas contemporáneos. Perspectivas clínicas, políticas, sociales y filosóficas (pp. 669-670). Buenos Aires: Asociación Argentina de Salud Mental.

Recibido: 2 de octubre de 2017 Aceptado: 26 de agosto de 2019 